Series A

I. MATHEMATICA

483

\title{
MINIMAL SURFACES WITH PARTIALLY FREE BOUNDARY
}

BY

JOHANNES C. C. NITSCHE

To Professor Lothar Collatz on his 60th birthday

H E L S I N K I I 97 l

S U OMALA INEN T I E D E A K A T E M I A

doi:10.5186/aasfm. 1971.483 
Copyright (C) 1971 by

Academia Scientiarum Fennica

Communicated 9 October 1970 by OLLI LeHto

Manuscript received 27 May 1970 


\section{Minimal surfaces with partially free boundary}

1. Consider a configuration in Euclidean 3-space consisting of a surface $T$ and of a rectifiable Jordan arc $\Gamma=\{\mathfrak{x}=\mathfrak{z}(t) ; 0 \leqq t \leqq \pi\}$ having its end points on $T$, but no other points in common with $T$. Denote by $P$ the semi-disc in the $(u, v)$-plane,

$$
P=\left\{u, v ; u^{2}+v^{2}<1, v>0\right\},
$$

by $\partial^{\prime} P$ and $\partial^{\prime \prime} P$ its boundary portions $\left\{u, v ; u^{2}+v^{2}=1, v>0\right\}$ and $\{u, v ;-1<u<1, v=0\}$, respectively, and by $P^{\prime}$ the domain $P \cup \partial^{\prime} P$.

A surface

$$
S=\left\{\mathfrak{x}=\mathfrak{x}(u, v) ;(u, v) \in P^{\prime}\right\}
$$

is said to be bounded by the configuration, or chain $\langle\Gamma, T\rangle$, if its position vector $\mathfrak{x}(u, v)=\{x(u, v), y(u, v), z(u, v)\}$ satisfies the following conditions:

i) $\mathfrak{x}(u, v) \in C^{0}\left(P^{\prime}\right)$.

ii) $\mathfrak{x}(u, v)$ maps the arc $\partial^{\prime} P$ onto the open arc

$$
\Gamma=\{\mathfrak{x}=\mathfrak{z}(t) ; 0<t<\pi\}
$$

monotonically in such a way that

$$
\lim _{\vartheta \rightarrow+0} \mathfrak{x}(\cos \vartheta, \sin \vartheta)=z(0), \quad \lim _{\vartheta \rightarrow \pi-0} \mathfrak{x}(\cos \vartheta, \sin \vartheta)=z(\pi) ;
$$

i.e. there exists a monotonously increasing continuous function $t=t(\vartheta)$, mapping the interval $0<\vartheta<\pi$ onto the interval $0<t<\pi$, such that $\mathfrak{x}(\cos \vartheta, \sin \vartheta)=\mathfrak{z}(t(\vartheta))$.

iii) The relation $\lim _{n \rightarrow \infty} d_{T}\left[\mathfrak{x}\left(u_{n}, v_{n}\right)\right]=0$ holds for every sequence of points $\left(u_{n}, v_{n}\right)$ in $P^{\prime}$ converging to a point on $\overline{\partial^{\prime \prime} P}$.

Here $d_{T}[\mathfrak{x}]=\inf _{\mathrm{t} \in T}|\mathfrak{x}-\mathfrak{t}|$ denotes the distance between the point $\mathfrak{x}$ and the surface $T$.

Obviously, the convergence specified under iii) is uniform in the following sense:

$$
\lim _{\delta \rightarrow 0} \sup _{\substack{u, v \in \in P^{\prime} \\ 0<v \leqq \delta}} d_{T}[\mathfrak{x}(u, v)]=0
$$


While thus the distance function $d_{T}[\mathfrak{x}(u, v)]$ is continuous in $\bar{P}$, the same cannot generally be said about the vector $\mathfrak{x}(u, v)$. In fact, the trace of $S$ on $T$, i.e. the set of limit points on $T$ for all sequences $\mathfrak{x}\left(u_{n}, v_{n}\right)$ as in iii) above, may well look quite bizarre. Examples illustrating such contingencies can be found in [4], pp. 95-96, and [6], pp. 220-222.

We shall denote by $\mathfrak{R}$ the collection of all surfaces

$$
S=\left\{\mathfrak{x}=\mathfrak{x}(u, v) ;(u, v) \in P^{\prime}\right\}
$$

bounded by the chain $\langle\Gamma, T\rangle$ and having finite (Lebesgue) area $A(S)$, and by $\mathfrak{A}$ the subclass of such surfaces whose position vector belongs to $C^{0}\left(P^{\prime}\right) \cap H_{2}^{1}(P)$. Here $H_{2}^{1}(P)$ is the pertinent Sobolev space insuring that $\mathfrak{x}(u, v)$ possesses square summable generalized first derivatives. On the strength of basic theorems by E. J. McShane [14] and C. B. Morrey [16][19] the area of a surface of class $\mathfrak{A}$ can be expressed by the classical area integral

$$
A(S)=\int_{P} \int_{P}\left|\mathfrak{x}_{u} \times \mathfrak{x}_{v}\right| d u d v
$$

Even if $S$ does not belong to $\mathfrak{A}$, this formula is correct, provided that the vector $\mathfrak{x}(u, v)$ belongs to $C^{0}\left(\overline{P^{(\delta)}}\right) \cap H_{2}^{1}\left(P^{(\delta)}\right)$ for each $\delta>0$, where

$$
P^{(\delta)}=\left\{u, v ; u^{2}+v^{2}<1, v>\sin \delta\right\},
$$

and that $\left|\mathfrak{x}_{u} \times \mathfrak{x}_{v}\right|$ is summable over $P$. These facts and all further information needed concerning the Lebesgue area can be found in the monographs by L. Cesari [2] and T. Radó [25].

If the end points of $\Gamma$ can be connected on $T$ by a rectifiable Jordan arc, then the solution of Plateau's problem for the resulting closed contour represents a surface of class $\mathfrak{A}$. In $1938 \mathrm{R}$. Courant proved that, whenever the class $\mathfrak{A}$ is not empty, there exists in $\mathfrak{A}$ a surface

$$
S=\left\{\mathfrak{x}=\mathfrak{x}(u, v) ;(u, v) \in P^{\prime}\right\}
$$

minimizing the value of Dirichlet's integral

$$
D_{P}[\mathfrak{x}]=\frac{1}{2} \iint_{P}\left(\mathfrak{x}_{u}^{2}+\mathfrak{x}_{v}^{2}\right) d u d v ;
$$

see [3], [4], pp. 87-96, [6], pp. 201-223, and for further results [7], [8], [26]. The position vector of the solution surface has the following additional properties:

$\left.i^{\prime}\right)$ The mapping of $\partial^{\prime} P$ onto $(T)$ is topological.

iv) $\mathfrak{x}(u, v)$ is harmonic in $P$ and satisfies in $P$ the conditions $\mathfrak{x}_{u}^{2}=\mathfrak{x}_{v}^{2}, \quad \mathfrak{x}_{u} \mathfrak{x}_{v}=0$. 
Naturally, there may be more than one solution.

Every one-to-one conformal transformation of $P$ onto itself, leaving the boundary portion $\partial^{\prime \prime} P$ invariant, leads to a new surface which is again bounded by the chain $\langle\Gamma, T\rangle$ and whose position vector has the same value of Dirichlet's integral. In order to remove this ambiguity and in order to guarantee that the convergence of the minimizing sequences on which the existence proof is based does not fail due to a degeneration of these conformal mappings, we shall stipulate as part of the definitions of the classes $\mathfrak{L}$ and $\mathfrak{A}$ that the vector $\mathfrak{x}(u, v)$ maps the point $(u, v)=$ $(0,1)$ of $\partial^{\prime} P$ onto the point $z(\pi / 2)$. From $\mathfrak{x}(\cos \vartheta, \sin \vartheta)=z(t(\vartheta))$ we then conclude that $t(\pi / 2)=\pi / 2$.

If $T$ is the boundary of a (bounded or unbounded) convex domain, and if $\Gamma$ lies entirely in this domain, then also the solution surface is contained in this domain. Generally, however, although the Jordan arc $\Gamma$ has only its end points in common with $T$, it is - even for the case of a convex surface with $\Gamma$ on the outside - unavoidable that $S$ permeates $T$. If one prefers to think of the boundary surface as an impenetrable object - as seems natural in view of the experiments - then one would be led to our investigation of the type initiated in [21]. Absolutely nothing has been done yet in this direction for the case at hand.

For the last three decades it has been a problem of great challenge to study the regularity of the solution surface on its free boundary and the nature of its trace. In this context the search for minimal conditions on the bounding surface $T$ which would guarantee the continuity of the trace deserves particular attention. It is also one of the main concerns of the present investigation.

If $T$ is a plane, then that part of the trace which corresponds to the open arc $\partial^{\prime \prime} P$ is an analytic curve, and the solution vector $\mathfrak{x}(u, v)$ permits an analytic extension across $\partial^{\prime \prime} P$. This has been proved by $\mathrm{R}$. Courant [6], pp. 218-220, and I. F. Ritter [26], p. 60. In 1951 H. Lewy [13] considered the case where $T$ is a closed orientable analytic surface and showed that, upon removal from the solution surface $S$ of a portion having vanishing area, a new surface $S^{*}$ of class $\mathfrak{A}$ can be obtained whose trace is an analytic curve. Further remarks and results are due to R. Courant [5] and S. Hildebrandt [10]. In a recent paper [11] W. Jäger discusses the problem for an orientable differential geometric surface of class $C^{2}$ which, if it is not compact, satisfies a certain uniformity condition. Using an interesting comparison procedure he shows that the position vector of a surface in $\mathfrak{A}$ which minimizes Dirichlet's integral has a Hölder continuous extension to each domain $\bar{P}_{r}$, where

$$
P_{r}=\left\{u, v ; u^{2}+v^{2}<r^{2}, v>0\right\}, \quad 0<r<1
$$


While the Hölder constant depends on $r$, the Hölder exponent can be chosen uniformly for all $r, 0<r<1$. As a matter of fact, a detailed analysis, given in [22], reveals that every exponent $\beta<1 / 2$ can be achieved in this way. Thus the part of the trace corresponding to the open arc $\partial^{\prime \prime} P$ is a continuous curve, each compact subarc of it being Hölder continuous, and, incidentally, Lewy's transition from $S$ to $S^{*}$ turns out to be unnecessary.

The methods which have been successful so far require, among other things, that the normal vector of the bounding surface $T$ have continuous first derivatives and thus fail to be applicable if $T$ does not possess a certain "starting regularity" $-C^{2}$ at least. If one wants to go further one has to free oneself from the use of a coordinate system in which the surfaces parallel to $T$ play the role of coordinate surfaces. In the developments to follow we shall show that the Hölder continuity of the trace can be established already for a bounding surface of class $C^{1}$. Postponing to paragraph 3 below the precise definition of an admissible boundary surface of class $C^{1}-$ closed (i.e. compact) regular surfaces of class $C^{1}$ imbedded in space are examples of such surfaces - we formulate our theorem as follows:

Theorem 1. If $T$ is an admissible boundary surface of class $C^{1}$, then the solution vector $\mathfrak{x}(u, v)$ (minimizing Dirichlet's integral in the class $\mathfrak{A}$ ) has a continuous extension to each domain $\bar{P}_{r}, 0<r<1$, belonging to class $C^{0, \gamma}\left(\bar{P}_{r}\right)$. Here $\gamma$ is an arbitrary positive number smaller than $1 /(\pi \sqrt{2})$ $=0.2248$.

The Hölder constant depends on $\gamma$ and $r$, on the geometric properties of the chain $\langle T, T\rangle$, and on the solution vector $\mathfrak{x}(u, v)$.

Our method of proof has to rely on a basic property of the solution surface - its »least area property» - which, surprisingly, seems to have escaped attention so far, although the corresponding, but easier to demonstrate, property of the classical solution for Plateau's problem is well documented; see [9], pp. 318-320, [24], pp. 90-95, [6], pp. 116-117. This property is expressed by the

Theorem 2. The area of a surface of class $\mathfrak{A}$ whose position vector minimizes Dirichlet's integral (in this class) simultaneously represents the minimum of the areas of all surfaces of class $\mathfrak{\Omega}$.

Paragraph 2 is devoted to the demonstration of theorem 2. Admissible boundary surfaces are defined in paragraph 3 , and their pertinent properties are discussed in paragraph 4. Paragraph 5 finally contains the proof of theorem 1 .

For simplicity's sake we shall avail ourselves also of the complex notation. Setting $w=u+i v=\varrho e^{i \vartheta}$ we shall interchangeably write $\mathfrak{x}(u, v)$, or $\mathfrak{x}(w)$, or $\mathfrak{x}(\varrho, \vartheta)$ - whichever is most convenient.

Statements about the end points of the trace are possible if the Jordan 
arc $T$ meets the surface $T$ properly. These questions, as well as further applications of our method, will be the subject of a subsequent investigation.

2. Let

$$
S=\left\{\mathfrak{x}=\mathfrak{y}(u, v) ;(u, v) \in P^{\prime}\right\}
$$

be a surface of class $\mathfrak{Z}$. For what follows we shall describe a construction leading from $S$ to a surface

$$
\bar{S}=\left\{\mathfrak{x}=\overline{\mathfrak{l}}(u, v) ;(u, v) \in P^{\prime}\right\}
$$

belonging to class $\mathfrak{A}$ for which $A(\bar{S}) \leqq D_{P}[\overline{\mathfrak{y}}] \leqq A(S)$. Theorem 2 will thus be demonstrated.

Let $\varepsilon_{1}>\varepsilon_{2}>\cdots$ be a sequence of positive numbers converging to zero and set

$$
S_{n}=S\left[\overline{P^{\left(\varepsilon_{n}\right)}}\right]=\left\{\mathfrak{x}=\mathfrak{l}(u, v) ;(u, v) \in \overline{P^{\left(\varepsilon_{n}\right)}}\right\} .
$$

Obviously $A\left(S_{n}\right) \leqq A(S)$. For fixed $n$ the surface $S_{n}$ can be approximated by a polyhedral surface $\Sigma_{n}$ with a Jordan domain as parameter set in such a way that the Fréchet distance $\left\|S_{n}, \Sigma_{n}\right\|$ satisfies the inequality $\left\|S_{n}, \Sigma_{n}\right\|<1 / n$ and that $A\left(\Sigma_{n}\right)<A\left(S_{n}\right)+1 / n$. By a fundamental mapping theorem already proved by H. A. Schwarz ([27]; see also [1], pp. 98-102) $\Sigma_{n}$ possesses a representation

$$
\left.\Sigma_{n}=\{\mathfrak{x}=\mathfrak{l})_{n}(u, v) ;(u, v) \in \bar{P}\right\}
$$

whose position vector is continuous in $\bar{P}$ and analytic in $P$ with the exception of the points on finitely many analytic ares subdividing $P$. In the points of analyticity the relations

$$
\left(\frac{\partial \mathfrak{y}_{n}}{\partial u}\right)^{2}=\left(\frac{\partial \mathfrak{y})_{n}}{\partial v}\right)^{2}, \quad \frac{\partial \mathfrak{y})_{n}}{\partial u} \frac{\partial \mathfrak{y})_{n}}{\partial v}=0
$$

are satisfied. Therefore $\mathfrak{y}_{n}(u, v)$ belongs to $C^{0}(\bar{P}) \cap H_{2}^{1}(P)$, and $A\left(\Sigma_{n}\right)=$ $D_{P}\left[\mathfrak{y}_{u}\right]$. We note that, due to the invariance of Dirichlet's integral, a change of parameters induced by a conformal mapping of $P$ onto itself is still permissible.

Since $\left\|S_{n}, \Sigma_{n}\right\|<1 / n$ there is a homeomorphism $\omega(w)$ of $\bar{P}$ onto $\overline{P^{\left(\varepsilon_{n}\right)}}$ such that $\left|\mathfrak{y}_{n}(w)-\mathfrak{y}(\omega(w))\right|<1 / n$ for $w \in \bar{P}$. Under this homeomorphism a certain connected subarc of $\partial P$ is mapped onto the arc

$$
\partial^{\prime} P^{\left(\varepsilon_{n}\right)}=\left\{u, v ; u^{2}+v^{2}=1, v>\sin \varepsilon_{n}\right\} .
$$

We now carry out a conformal (or, if necessary, anti-conformal) mapping of the semi-disc $P$ onto itself which is so chosen that the above subarc 
goes over into the arc $\partial^{\prime} P$ in such a way that the points $w=1, w=i$, and $w=-1$ correspond to the points $e^{i \varepsilon_{n}}, i, e^{i\left(\tau-\varepsilon_{n}\right)}$, respectively, of $\partial^{\prime} P^{\left(\varepsilon_{n}\right)}$. After this transformation there is a monotonously increasing continuous function $\tau=\tau_{n}(\vartheta)$ which maps the interval $0 \leqq \vartheta \leqq \pi$ onto the interval $\varepsilon_{n} \leqq \tau \leqq \pi-\varepsilon_{n}$ so that

$$
\left|\mathfrak{y} n\left(e^{i \vartheta}\right)-\mathfrak{y}\left(e^{i_{\tau_{n}}(\vartheta)}\right)\right|=\left|\mathfrak{y}_{n}\left(e^{i \vartheta}\right)-\mathfrak{z}\left(t_{n}(\vartheta)\right)\right|<\frac{1}{n}
$$

for $0 \leqq \vartheta \leqq \pi$. Here we have set $t_{n}(\vartheta)=t\left(\tau_{n}(\vartheta)\right)$. The monotonously increasing continuous function $t_{n}(\vartheta)$ maps the interval $0 \leqq \vartheta \leqq \pi$ onto the interval $\varepsilon_{n}^{\prime} \equiv t_{n}\left(\varepsilon_{n}\right) \leqq t \leqq t_{n}\left(\pi-\varepsilon_{n}\right) \equiv \pi-\varepsilon_{n}^{\prime \prime}$. It is possible that $\varepsilon_{n}^{\prime}=0$ or $\varepsilon_{n}^{\prime \prime}=0$. In any event, however, $\varepsilon_{1}^{\prime} \geqq \varepsilon_{2}^{\prime} \geqq \cdots$ and $\varepsilon_{1}^{\prime \prime} \geqq \varepsilon_{2}^{\prime \prime} \geqq \cdots$. Moreover, if we set

we have

$$
d_{n}=\sup _{\substack{(u, v) \in P^{\prime} \\ 0<v<\sin \varepsilon_{n}}} d_{T}[\mathfrak{y}(u, v)]
$$

$$
d_{T}\left[\mathfrak{y}_{n}(w)\right] \leqq d_{T}[\mathfrak{y}(\omega(w))]+\left|\mathfrak{y}_{n}(w)-\mathfrak{y}(\omega(w))\right| \leqq d_{n}+\frac{1}{n} \quad \text { for } \quad w \in \overline{\partial^{\prime \prime} P} .
$$

Of course, $\lim _{n \rightarrow \infty} d_{n}=0$.

Having determined the polyhedral surfaces $\Sigma_{1}, \Sigma_{2}, \cdots$ and having obtained the monotone functions $t_{1}(\vartheta), t_{2}(\vartheta), \cdots$, we observe that Helly's selection principle guarantees the existence of a subsequence $\left\{t_{n_{j}}(\vartheta)\right\}$ converging for $0 \leqq \vartheta \leqq \tau$ to a monotone limit function $t(\vartheta)$. For simplicity's sake this subsequence will again be denoted by $\left\{t_{n}(\vartheta)\right\}$. For a fixed $\vartheta$ in $0<\vartheta<\pi$ then

$$
\lim _{n \rightarrow \infty} \mathfrak{y}_{n}\left(e^{i \vartheta}\right)=\lim _{n \rightarrow \infty} \mathfrak{z}\left(t_{n}(\vartheta)\right)=\mathfrak{z}(t(\vartheta)) .
$$

Let $\vartheta_{0}$ be a fixed angle in $0<\vartheta_{0}<\tau$ and assume that the limits $t\left(\vartheta_{0}-0\right)$ and $t\left(\vartheta_{0}+0\right)$ are different so that $0 \leqq t\left(\vartheta_{0}-0\right)<t\left(\vartheta_{0}+0\right) \leqq \pi$. Since the vector $z(t)$ provides a topological representation of $\Gamma$, then $z\left(t\left(\vartheta_{0}-0\right)\right)+z\left(t\left(\vartheta_{0}+0\right)\right)$. Remembering that $D_{P}\left[\mathfrak{l}_{n}\right] \leqq A(S)+1$ for $n=$ $1,2, \cdots$, a well-known argument (see for instance [15], pp. 722-724) now leads to a contradiction. Thus we see that the limit function $t(\vartheta)$ is continuous in $0<\vartheta<\pi$. Under these circumstances (see [23], Aufgabe 127 on p. 62) the convergence $t_{n}(\vartheta) \rightarrow t(\vartheta)$ must be uniform in every closed subinterval of $0<\vartheta<\pi$.

We now assert that $t(+0)=0$ and $t(\tau-0)=\tau$. For the proof of the first limit relation assume that, on the contrary, $t(+0)=t_{0}>0$, but certainly $t_{0} \leqq \pi / 2$ since $t_{n}(\pi / 2)=\pi / 2$ for $n=1,2, \cdots$. Then $d_{T}\left[z\left(t_{0}\right)\right]$ $>0$ and therefore $d_{T}[z(t(\vartheta))] \geqq \mu>0$ for $0<\vartheta<\pi / 2$. The circle 
of radius $\delta, 0<\delta<1$, intersects the boundary $\partial P$ in two points $w_{1}(\delta)=1-\delta \quad$ and $\quad w_{2}(\delta)=e^{i \vartheta(\delta)}$, where $\vartheta(\delta)=2 \arcsin (\delta / 2)$. An argument going back to $H$. Lebesgue ([12], p. 388; see also [6], pp. 11, $101-103$, and [28], pp. 331-332) now tells us the following: For every $\delta$, $0<\delta<1$, and every $n=1,2, \cdots$ there is a number $\delta_{n}$ in $\delta \leqq$ $\delta_{n} \leqq \sqrt{\delta}$ such that

$$
\left.\mid \mathfrak{y})_{n}\left(1-\delta_{n}\right)-\mathfrak{y}\right)_{n}\left(e^{i \vartheta_{n}}\right) \mid \leqq \frac{M}{\log \frac{1}{\delta}}, \quad M=[2 \pi(A(S)+1)]^{1 / 2} .
$$

Here $\vartheta(\delta) \leqq \vartheta_{n} \equiv \vartheta\left(\delta_{n}\right) \leqq \vartheta(\sqrt{ } \delta)$. Combining our inequalities we find

$$
\begin{aligned}
\mu \leqq & d_{T}\left[z\left(t\left(\vartheta_{n}\right)\right)\right] \\
\leqq & d_{T}\left[\mathfrak{y}_{n}\left(1-\delta_{n}\right)\right]+\left|\mathfrak{y}_{n}\left(1-\delta_{n}\right)-\mathfrak{y}_{n}\left(e^{i \vartheta_{n}}\right)\right| \\
& +\left|\mathfrak{y}{ }_{n}\left(e^{i \vartheta_{n}}\right)-\mathfrak{z}\left(t_{n}\left(\vartheta_{n}\right)\right)\right|+\left|z\left(t_{n}\left(\vartheta_{n}\right)\right)-\mathfrak{z}\left(t\left(\vartheta_{n}\right)\right)\right| \\
\leqq & d_{n}+\frac{1}{n}+\frac{M}{\log \frac{1}{\delta}}+\frac{1}{n}+\left|z\left(t_{n}\left(\vartheta_{n}\right)\right)-\mathfrak{z}\left(t\left(\vartheta_{n}\right)\right)\right|
\end{aligned}
$$

or, for $\delta=\exp (-2 M / \mu)$,

$$
\mu \leqq 2 d_{n}+\frac{4}{n}+z\left(t_{n}\left(\vartheta_{n}\right)\right)-z\left(t\left(\vartheta_{n}\right)\right) \mid
$$

In view of the uniform convergence $t_{n}(\vartheta) \rightarrow t(\vartheta)$ in compact subintervals of $0<\vartheta<\pi$ and the fact that $\vartheta(\delta) \leqq \vartheta_{n} \leqq \vartheta(\sqrt{\delta})$ for $n=1,2, \cdots$ we arrive at a contradiction. The relation $t(\tau-0)=\pi$ is proved in the same way.

Denote by $\overline{\mathfrak{y}}_{n}(w)$ the vector which is harmonic in $P$, continuous in $\bar{P}$, and has on $\partial P$ the same values as $\mathfrak{y}_{n}(u)$. We summarize the properties of $\overline{\mathfrak{y}}_{n}(w)$ :

$$
\left|\overline{\mathfrak{y}}_{n}\left(e^{i \vartheta}\right)-\mathfrak{z}\left(t_{n}(\vartheta)\right)\right|<\frac{1}{n} \quad \text { for } \quad 0<\vartheta<\tau \text { and } n=1,2, \cdots .
$$

The monotone function $t(\vartheta)$ is continuous in $0<\vartheta<\pi$ and has the limits $t(+0)=0, \quad t(\pi-0)=\pi$. The convergence $t_{n}(\vartheta) \rightarrow t(\vartheta)$ is uniform in every compact subinterval of $0<\vartheta<\pi$. Moreover, by Dirichlet's principle,

$$
D_{P}\left[\overline{\mathfrak{y}}_{n}\right] \leqq D_{P}\left[\mathfrak{y}{ }_{n}\right] \leqq A(S)+\frac{1}{n}
$$


The remainder of the proof of theorem 2 consists in the step-by-step demonstration of the following facts:

1) The vectors $\overline{\mathfrak{y}}_{n}(w)$ are uniformly bounded in every compact subset of $P$. This follows from the uniform boundedness of the Dirichlet integral in conjunction with the fact that

$$
\begin{aligned}
\left|\overline{\mathfrak{y}}_{n}\left(e^{i \vartheta}\right)\right| & \leqq\left|\overline{\mathfrak{y}}_{n}\left(e^{i \vartheta}\right)-z\left(t_{n}(\vartheta)\right)\right|+\left|z\left(t_{n}(\vartheta)\right)\right| \\
& \leqq \frac{1}{n}+\left|z\left(t_{n}(\vartheta)\right)\right| \\
& \leqq 1+\max _{0 \leqq i \leqq \tau}|z(t)| .
\end{aligned}
$$

2) A subsequence of the $\overline{\mathfrak{y}}_{n}(w)$ - we call it at once again $\left\{\overline{\mathfrak{y}}_{n}(w)\right\}$ - converges in $P$ to a harmonic vector $\overline{\mathfrak{y}}(w)$. The convergence is uniform in every compact subdomain of $P$. This is a consequence of Harnack's theorem.

3) The sequence $\left\{\overline{\mathfrak{y}}_{n}(w)\right\}$ converges on $\partial^{\prime} P$ to a limit vector. The convergence is uniform on every compact subarc of $\partial^{\prime} P$. This follows from the uniform convergence $t_{n}(\vartheta) \rightarrow t(\vartheta)$.

4) A subsequence of the previous sequence - again denoted by $\left\{\overline{\mathfrak{y}}_{n}(w)\right\}$ - converges uniformly in every compact subset of $P^{\prime}=P \cap \partial^{\prime} P$. The limit vector, of course, is $\overline{\mathfrak{y}}(w)$. This can be proved using a classical device due to $H$. Lebesgue; see [12], pp. 386-388.

5) If $Q$ is a compact subdomain of $P$, we have

$$
D_{Q}[\overline{\mathfrak{y}}]=\lim _{n \rightarrow \infty} D_{Q}\left[\overline{\mathfrak{y}}_{n}\right] \leqq \liminf _{n \rightarrow \infty} D_{P}\left[\overline{\mathfrak{y}}_{n}\right] \leqq A(S) .
$$

Thus $D_{P}[\overline{\mathfrak{y}}] \leqq A(S)$.

6) If $\left\{w_{j}\right\}$ is a sequence of points in $P^{\prime}$ converging to a point on $\partial^{\prime \prime} P$, then

$$
\lim _{j \rightarrow \infty} d_{T}\left[\overline{\mathfrak{y}}\left(w_{j}\right)\right]=0 .
$$

This is demonstrated by a method of R. Courant: see [4], pp. 89-91, and [6], pp. 203-205.

We have now established that $\overline{\mathfrak{y}}(w)$ is the position rector of a surface $\bar{S}=\left\{\mathfrak{x}=\overline{\mathfrak{y}}(w) ; w \in P^{\prime}\right\}$ of class $\mathfrak{A}$ which is bounded by the chain $\langle\Gamma, T\rangle$ and that $A(\bar{S}) \leqq A(S)$. Theorem 2 is proved.

3. Let $T$ be a differential geometric regular surface of class $C^{1}$. Consider an interior point $\mathrm{t}_{0}$ of $T$, and choose a coordinate system in which $\mathrm{t}_{0}$ becomes the point $(1,0,0)$ and the plane $x=1$ the tangent plane to $T$ at $\mathrm{t}_{0}$. Then $T$ has a local representation $x=f(y, z)$. The function 
$f(y, z)$ is continuously differentiable and satisfies the relations $f(0,0)=1$, $f_{y}(0,0)=f_{z}(0,0)=0$. We now introduce spherical coordinates $(r, \alpha, \beta)$ in the usual way setting

$$
x=r \sin \alpha \cos \beta, \quad y=r \sin \alpha \sin \beta, \quad z=\cos \alpha .
$$

Provided the number $\delta, 0<\delta<1$, is chosen small enough, the connected portion of $T$ containing the point $(1,0,0)$ and lying within the spatial cone defined by $|\alpha-\pi / 2|<\delta, \quad|\beta|<\delta$ has a representation $\mathfrak{x}=$ $R^{*}(\alpha, \beta) \mathfrak{R}^{*}(\alpha, \beta)$. Here $\mathfrak{R}^{*}(\alpha, \beta)$ denotes the unit vector

$$
\mathfrak{R}^{*}(\alpha, \beta)=\{\sin \alpha \cos \beta, \sin \alpha \sin \beta, \cos \alpha\} .
$$

$R^{*}(\alpha, \beta)$ is a continuously differentiable function satisfying $R^{*}(\pi / 2,0)$ $=1$. It can be obtained by elimination from the identity

$$
F\left(\alpha, \beta, R^{*}\right) \equiv R^{*} \sin \alpha \cos \beta-f\left(R^{*} \sin \alpha \sin \beta, R^{*} \cos \alpha\right)=0
$$

for which $F_{R^{*}}(\pi / 2,0,1)=1$. A simple computation shows that

$$
R_{\alpha}^{* 2}+\frac{1}{\sin ^{2} \alpha} R_{\beta}^{* 2}=0 \quad \text { for } \quad \alpha=\frac{\pi}{2}, \beta=0 .
$$

Therefore, given any positive number $c, \delta$ can be chosen so small that

$$
R_{\alpha}^{* 2}(\alpha, \beta)+\frac{1}{\sin ^{2} \alpha} R_{\beta}^{* 2}(\alpha, \beta)<c^{2} \quad \text { for }\left|\alpha-\frac{\pi}{2}\right|<\delta,|\beta|<\delta .
$$

We now set $\varepsilon=\sin \delta$ and consider the three-dimensional region $B=$ $B\left(\mathrm{t}_{0}, c ; \varepsilon\right)$ defined by the inequalities

$$
1-\varepsilon<r<1+\varepsilon, \quad\left|\alpha-\frac{\pi}{2}\right|<\arcsin \varepsilon, \quad|\beta|<\arcsin \varepsilon .
$$

This region contains the ball $(x-1)^{2}+y^{2}+z^{2}<\varepsilon^{2}$, and we can use in it the spherical coordinates $r, \alpha, \beta$ instead of the Cartesian coordinates $x, y, z$. For the inverse functions $r(x, y, z), \quad x(x, y, z), \beta(x, y, z)$ we have

$$
\begin{aligned}
\operatorname{grad} r(x, y, z) & =\mathfrak{N}^{*}(\alpha, \beta) \\
\operatorname{grad} \alpha(x, y, z) & =\frac{1}{r} \mathfrak{N}_{\alpha}^{*}(\alpha, \beta), \\
\operatorname{grad} \beta(x, y, z) & =\frac{1}{r \sin ^{2} \alpha} \mathfrak{N}_{\beta}^{*}(\alpha, \beta) .
\end{aligned}
$$
form

Every point $\mathfrak{x}=\{x, y, z\}$ in $B$ has a unique representation of the 


$$
\mathfrak{x}=[R(\mathfrak{x})+\lambda(\mathfrak{x})] \mathfrak{N}(\mathfrak{x})
$$

where

$$
\begin{aligned}
R(\mathfrak{x})= & \left.R^{*}(\alpha(x, y, z)), \beta(x, y, z)\right), \\
\mathfrak{X}(\mathfrak{X})= & \mathfrak{l}^{*}(\alpha(x, y, z), \beta(x, y, z)), \\
& \lambda(\mathfrak{x})=|\mathfrak{x}|-R(\mathfrak{X})
\end{aligned}
$$

are continuously differentiable.

We have $R_{x}=R_{\alpha}^{*} \alpha_{x}+R_{\beta}^{*} \beta_{x}$ etc. and therefore

$$
\begin{aligned}
R_{x}^{2}+R_{y}^{2}+R_{z}^{2} & \leqq\left(R_{\alpha}^{* 2}+\frac{1}{\sin ^{2} \alpha} R_{\beta}^{* 2}\right)\left((\operatorname{grad} \alpha)^{2}+\sin ^{2} \alpha(\operatorname{grad} \beta)^{2}\right) \\
& \leqq 2 c^{2}|\mathfrak{x}|^{-2} \leqq 2 c^{2}(1-\varepsilon)^{-2}
\end{aligned}
$$

Given the number $c$ - for reasons to become clear later we shall always choose $0<c<1 / 2$ - every interior point $\mathfrak{t}$ of $T$ is for a suitable $\varepsilon$, $0<\varepsilon<1$, the center of a domain $B(\mathrm{t}, c ; \varepsilon)$ in which the above inequalities for the functions $R^{*}(\alpha, \beta)$ and $R(\mathfrak{x})$ are valid. The special coordinate system introduced in the definition of $B(\mathrm{t}, c ; \varepsilon)$ will be called a distinguished coordinate system associated with the point $t$.

If $T$ is a closed (i.e. compact) differential geometric regular surface imbedded in space, then the number $\varepsilon$ can be chosen uniformly for all points of $T$. As a matter of fact, this property is the precise condition of admissibility for the surface $T$.

Definition. The surface $T$ is an admissible boundary surface of class $C^{1}$ if it has the following properties:

i) For every point $\mathrm{t}$ of $T$ there $i$ s an open sphere $K$ containing $\mathfrak{t}$ and a function $g(\mathfrak{x}) \equiv g(x, y, z) \in C^{1}(K)$ with non-vanishing gradient such that the statements $\mathfrak{x} \in K, g(\mathfrak{x})=0$ and $\mathfrak{x} \in T \cap K$ are equivalent.

ii) Given any number $c>0$, there is a number $\varepsilon=\varepsilon(c), 0<\varepsilon<1$ - the same for all points of $T$ - such that every point $\mathrm{t}$ of $T$ is the center of a region $B(\mathrm{t}, c ; \varepsilon)$ as defined before. In a distinguished coordinate system associated with $\mathrm{t}$ the connected part of $T$ containing $\mathfrak{t}$ and contained in the cone defined by $|\alpha-\pi / 2|<\arcsin \varepsilon,|\beta|<$ arc sin $\varepsilon$ has the representation $\mathfrak{x}=R^{*}(\alpha, \beta) \mathfrak{l}^{*}(\alpha, \beta)$ where

$$
R_{\alpha}^{*_{2}}+\frac{1}{\sin ^{2} \alpha} R_{\beta}^{*_{2}} \leqq c^{2} .
$$

Every point $\mathfrak{x}$ in $B(\mathfrak{t}, c ; \varepsilon)$, in particular every point in the ball $|\mathfrak{x}-\mathfrak{t}|<\varepsilon$, has the representation $\mathfrak{x}=[R(\mathfrak{c})+\lambda(\mathfrak{x})] \mathfrak{l}(\mathfrak{x})$ in which $R(\mathfrak{x}), \lambda(\mathfrak{x})$ and $\mathfrak{N}(\mathfrak{x})$ are continuously differentiable. 
4. Let $T$ be an admissible boundary surface of class $C^{1}$ and consider a vector $\mathfrak{x}(u, v)$ which has continuous first derivatives in a domain $Q$, satisfying there the relations $\mathfrak{x}_{u}^{2}=\mathfrak{x}_{v}^{2}, \mathfrak{x}_{u} \mathfrak{x}_{v}=0$ and mapping $Q$ into a domain $B(\mathrm{t}, c ; \varepsilon)$. After subjecting the vector $\mathfrak{x}(u, v)$ to an orthogonal transformation with constant elements we may work in a distinguished coordinate system associated with the point $\mathfrak{t}$. Using the abbreviations $R(u, v)=R(\mathfrak{x}(u, v))$ etc. we have

$$
\mathfrak{x}(u, v)=[R(u, v)+\lambda(u, v)] \mathfrak{N}(u, v) .
$$

We also set $h(u, v)=4\left(\mathfrak{x}_{u}^{2}+\mathfrak{x}_{v}^{2}\right)^{-2}\left(\mathfrak{x}_{u}, \mathfrak{x}_{v}, \mathfrak{R}\right)^{2}$ so that $0 \leqq h(u, v) \leqq 1$.

Lemma. The following inequalities hold:

$$
\begin{aligned}
& \lambda_{u}^{2}+\lambda_{v}^{2} \leqq \frac{1}{2}\left(\sqrt{1-h}+\frac{2 c}{|\mathfrak{x}|}\right)^{2}\left(\mathfrak{x}_{u}^{2}+\mathfrak{x}_{v}^{2}\right), \\
& \mathfrak{R}_{u}^{2}+\mathfrak{N}_{v}^{2} \leqq \frac{1}{2} \frac{1+h}{|\mathfrak{x}|^{2}}\left(\mathfrak{x}_{u}^{2}+\mathfrak{x}_{v}^{2}\right) .
\end{aligned}
$$

Proof. Since the vector $\mathfrak{R}$ is orthogonal to $\mathfrak{R}_{u}$ and $\mathfrak{R}_{v}$, we find

$$
\begin{aligned}
\mathfrak{X}_{u}^{2} & =\left(R_{u}+\lambda_{u}\right)^{2}+(R+\lambda)^{2} \mathfrak{N}_{u}^{2}, \\
\mathfrak{X}_{v}^{2} & =\left(R_{v}+\lambda_{v}\right)^{2}+(R+\lambda)^{2} \mathfrak{R}_{v}^{2}, \\
\mathfrak{x}_{u} \mathfrak{C}_{v} & =\left(R_{u}+\lambda_{u}\right)\left(R_{v}+\lambda_{v}\right)+(R+\lambda)^{2} \mathfrak{N}_{u} \mathfrak{P}_{v} .
\end{aligned}
$$

The relations $\mathfrak{x}_{u}^{2}=\mathfrak{x}_{v}^{2}, \mathfrak{x}_{u} \mathfrak{x}_{v}=0$ imply

$$
\begin{aligned}
{\left[\left(R_{u}+\lambda_{u}\right)^{2}+\left(R_{v}+\lambda_{v}\right)^{2}\right]^{2} } & =\left[\left(R_{u}+\lambda_{u}\right)^{2}-\left(R_{v}+\lambda_{v}\right)^{2}\right]^{2}+4\left(R_{u}+\lambda_{u}\right)^{2}\left(R_{v}+\lambda_{v}\right)^{2} \\
& =(R+\lambda)^{4}\left[\left(\mathfrak{N}_{u}^{2}-\mathfrak{N}_{v}^{2}\right)^{2}+4\left(\mathfrak{N}_{u} \mathfrak{N}_{v}\right)^{2}\right] \\
& =(R+\lambda)^{4}\left[\left(\mathfrak{N}_{u}^{2}+\mathfrak{N}_{v}^{2}\right)^{2}-4\left(\mathfrak{N}_{u} \times \mathfrak{N}_{v}\right)^{2}\right]
\end{aligned}
$$

Now

$$
\begin{aligned}
\mathfrak{N}_{u} \times \mathfrak{N}_{v} & =\left(\alpha_{u} \mathfrak{N}_{\alpha}^{*}+\beta_{u} \mathfrak{N}_{\beta}^{*}\right) \times\left(\alpha_{v} \mathfrak{N}_{\alpha}^{*}+\beta_{v} \mathfrak{N}_{\beta}^{*}\right) \\
& =\left(\alpha_{u} \beta_{v}-\alpha_{v} \beta_{u}\right) \mathfrak{N}_{\alpha}^{*} \times \mathfrak{N}_{\beta}^{*} \\
& =\left(\alpha_{u} \beta_{v}-\alpha_{v} \beta_{u}\right) \sin \alpha \mathfrak{N}
\end{aligned}
$$

and

$$
\begin{aligned}
\alpha_{u} \beta_{v}-\alpha_{v} \beta_{u} & =\frac{\partial(\alpha, \beta)}{\partial(y, z)} \frac{\partial(y, z)}{\partial(u, v)}+\frac{\partial(x, \beta)}{\partial(z, x)} \frac{\partial(z, x)}{\partial(u, v)}+\frac{\partial(\alpha, \beta)}{\partial(x, y)} \frac{\partial(x, y)}{\partial(u, v)} \\
& =\frac{1}{r^{2}}\left[\cos \beta \frac{\partial(y, z)}{\partial(u, v)}+\sin \beta \frac{\partial(z, x)}{\partial(u, v)}+\frac{\cos \alpha}{\sin \alpha} \frac{\partial(x, y)}{\partial(u, v)}\right] \\
& =\frac{1}{|\mathfrak{x}|^{2} \sin \alpha}\left(\mathfrak{x}_{u}, \mathfrak{x}_{v}, \mathfrak{R}\right) .
\end{aligned}
$$


A. I. 483

Therefore

$$
\begin{aligned}
{\left[\left(R_{u}+\lambda_{u}\right)^{2}+\left(R_{v}+\lambda_{v}\right)^{2}\right]^{2} } & =(R+\lambda)^{4}\left[\left(\mathfrak{N}_{u}^{2}+\mathfrak{N}_{v}^{2}\right)^{2}-4|\mathfrak{x}|^{-4}\left(\mathfrak{X}_{u}, \mathfrak{X}_{v}, \mathfrak{R}\right)^{2}\right] \\
& =(R+\lambda)^{4}\left(\mathfrak{N}_{u}^{2}+\mathfrak{N}_{v}^{2}\right)^{2}-4\left(\mathfrak{C}_{u}, \mathfrak{X} v, \mathfrak{N}\right)^{2} .
\end{aligned}
$$

Substitution into the identity

$$
\mathfrak{x}_{u}^{2}+\mathfrak{x}_{v}^{2}=\left(R_{u}+\lambda_{u}\right)^{2}+\left(R_{v}+\lambda_{v}\right)^{2}+(R+\lambda)^{2}\left(\mathfrak{N}_{u}^{2}+\mathfrak{N}_{v}^{2}\right)
$$

leads to the following two equations:

$$
\begin{aligned}
\left(R_{u}+\lambda_{u}\right)^{2}+\left(R_{v}+\lambda_{v}\right)^{2} & =\frac{1}{2}(1-h)\left(\mathfrak{x}_{u}^{2}+\mathfrak{x}_{v}^{2}\right) \\
\mathfrak{N}_{u}^{2}+\mathfrak{N}_{v}^{2} & =\frac{1}{2} \frac{1+h}{|\mathfrak{x}|^{2}}\left(\mathfrak{x}_{u}^{2}+\mathfrak{x}_{v}^{2}\right) .
\end{aligned}
$$

From

$$
2\left|R_{u} \lambda_{u}+R_{v} \lambda_{v}\right| \leqq \sigma\left(\lambda_{u}^{2}+\lambda_{v}^{2}\right)+\frac{1}{\sigma}\left(R_{u}^{2}+R_{v}^{2}\right), \quad 0<\sigma<1,
$$

and

$$
R_{u}^{2}+R_{v}^{2} \leqq\left(R_{x}^{2}+R_{y}^{2}+R_{z}^{2}\right)\left(\mathfrak{x}_{u}^{2}+\mathfrak{x}_{v}^{2}\right) \leqq \frac{2 c^{2}}{|\mathfrak{x}|^{2}}\left(\mathfrak{x}_{u}^{2}+\mathfrak{x}_{v}^{2}\right)
$$

we find

$$
\begin{aligned}
(1-\sigma)\left(\lambda_{u}^{2}+\lambda_{v}^{2}\right) & \leqq \frac{1}{2}(1-h)\left(\mathfrak{x}_{u}^{2}+\mathfrak{x}_{v}^{2}\right)+\left(\frac{1}{\sigma}-1\right)\left(R_{u}^{2}+R_{v}^{2}\right) \\
& \leqq \frac{1}{2}\left[(1-h)+\frac{1-\sigma}{\sigma} \frac{4 c^{2}}{\mathfrak{x}^{2}}\right]\left(\mathfrak{x}_{u}^{2}+\mathfrak{x}_{v}^{2}\right)
\end{aligned}
$$

or

$$
\lambda_{u}^{2}+\lambda_{v}^{2} \leqq \frac{1}{2}\left[\frac{1-h}{1-\sigma}+\frac{4 c^{2}}{\sigma|\mathfrak{x}|^{2}}\right]\left(\mathfrak{x}_{u}^{2}+\mathfrak{x}_{v}^{2}\right)
$$

With the choice $\sigma=2 c /(2 c+|\mathfrak{x}| \sqrt{1-h})$ we finally obtain

$$
\lambda_{u}^{2}+\lambda_{v}^{2} \leqq \frac{1}{2}\left(\sqrt{1-h}+\frac{2 c}{|\mathfrak{x}|}\right)^{2}\left(\mathfrak{x}_{u}^{2}+\mathfrak{x}_{v}^{2}\right)
$$

The lemma is proved.

5. Let $C\left(w_{0} ; \varrho\right)$ denote the domain $\left\{w ; w \in P,\left|w-w_{0}\right|<\varrho\right\}$ and introduce the abbreviation 


$$
\left.D\left[\mathfrak{x} ; w_{0}, \varrho\right]=\frac{1}{2} \int_{\mathcal{C}\left(w_{0} ; \varrho\right)} \int_{\mathfrak{C}_{u}^{2}}+\mathfrak{x}_{v}^{2}\right) d u d v .
$$

In view of a well-known lemma of C. B. Morrey ([20], pp. 134-135) theorem 1 is a consequence of the following assertion:

Let $\gamma$ and $r$ be arbitrary numbers subject to the restrictions $0<\gamma<$ $1 /(\pi \sqrt{2}), 0<r<1$. Then there are positive constants $\delta<1$ and $M$ such that

$$
D\left[\mathfrak{x} ; w_{0}, \delta\right] \leqq M \varrho^{2 \gamma} \quad \text { for all } w_{0} \in P_{r}, \quad 0<\varrho<\delta .
$$

We proceed to prove this assertion. Let

$$
c=\frac{1}{2} \frac{1-\gamma \pi \sqrt{2}}{1+\gamma \pi \sqrt{2}} \quad \text { so that } \quad \frac{1}{\pi \sqrt{2}} \frac{1}{1+2 c}=\frac{1}{2}\left(\gamma+\frac{1}{\pi \sqrt{2}}\right)>\gamma,
$$

and choose $\varepsilon$ so small that $\varepsilon \leqq \varepsilon(c)$ and

$$
\frac{1-\varepsilon}{1+\varepsilon} \geqq \gamma \pi \sqrt{2}\left(1+\frac{2 c}{1-\varepsilon}\right) \text {. }
$$

Here $\varepsilon(c)$ is the number appearing in the definition of the admissible boundary surface $T$. Select a number $\delta, 0<\delta<(1-r) / 2$, so that

$$
\frac{1}{2} \int_{\substack{u^{2}+v^{2}<1 \\ 0<v<2 \delta}}\left(\mathfrak{x}_{u}^{2}+\mathfrak{x}_{v}^{2}\right) d u d v<\frac{1}{\sqrt{2}} \varepsilon^{2}
$$

Now consider a point $w_{0}=u_{0}+i v_{0}$ in $P_{r}$. For $v_{0} \geqq \delta$ and $0<\varrho<\delta$ we have

$$
D\left[\mathfrak{x} ; w_{0}, \varrho\right] \leqq\left(\frac{\varrho}{\delta}\right)^{2} D\left[\mathfrak{x} ; w_{0}, \delta\right] \leqq\left(\frac{\varrho}{\delta}\right)^{2_{\gamma}} D_{P}[\mathfrak{x}] .
$$

Here the first inequality follows simply with the help of the Fourier expansion for the vector $\mathfrak{x}$ (using polar coordinates $(\varrho, \vartheta)$ about $w_{0}$ )

$$
\mathfrak{x}(\varrho, \vartheta)=\frac{1}{2} \mathfrak{a}_{0}+\sum_{n=1}^{\infty} \varrho^{n}\left(\mathfrak{a}_{n} \cos n \vartheta+\mathfrak{b}_{n} \sin n \vartheta\right)
$$

from which

$$
\begin{aligned}
D\left[\mathfrak{x} ; w_{0}, \varrho\right] & =\frac{\pi}{2} \sum_{n=1}^{\infty} n \varrho^{2^{n}}\left(\mathfrak{a}_{n}^{2}+\mathfrak{b}_{n}^{2}\right) \\
& \leqq\left(\frac{\varrho}{\delta}\right)^{2} \frac{\pi}{2} \sum_{n=1}^{\infty} n \delta^{2 n}\left(\mathfrak{a}_{n}^{2}+\mathfrak{b}_{n}^{2}\right)=\left(\frac{\varrho}{\delta}\right)^{2} D\left[\mathfrak{x} ; w_{0}, \delta\right] .
\end{aligned}
$$


Assuming now that $0<v_{0}<\delta$ and $v_{0}<\varrho<\delta$, we have

$$
D\left[\mathfrak{x} ; w_{0}, \varrho\right] \leqq D\left[\mathfrak{x} ; u_{0}, v_{0}+\varrho\right] \equiv \Phi\left(v_{0}+\varrho\right),
$$

where we have set

$$
\Phi(r)=\frac{1}{2} \int_{0}^{r} d \varrho \int_{0}^{\pi}|\nabla \mathfrak{x}(\varrho, \vartheta)|^{2} \varrho d \vartheta,
$$

now using polar coordinates about the point $w=u_{0}$ on $\partial^{\prime \prime} P$ and where

$$
|\nabla \mathfrak{x}(\varrho, \vartheta)|^{2}=\mathfrak{x}_{\varrho}^{2}+\frac{1}{\varrho^{2}} \mathfrak{x}_{\vartheta}^{2} .
$$

Since $D_{P}[\mathfrak{x}]<\infty$, the derivative $\Phi^{\prime}(r)$ exists for almost all $r$ in $0<r$ $<2 \delta$ and is equal to

$$
\Phi^{\prime}(r)=\frac{1}{2} \int_{0}^{\pi}|\nabla \mathfrak{x}(r, \vartheta)|^{2} r d \vartheta .
$$

Our next aim is to show that the function $\Phi(r)$ satisfies for almost all $r$ in $v_{0}<r<v_{0}+\delta$ the differential inequality

$$
2 \gamma \Phi(r) \leqq r \Phi^{\prime}(r)
$$

For the proof by contradiction consider a value $r$ in $v_{0}<r<v_{0}+\delta$ for which $\Phi^{\prime}(r)$ exists and $r \Phi^{\prime}(r)<2 \gamma \Phi(r)$. For $0<\vartheta^{\prime}<\vartheta^{\prime \prime}<\pi$ we then have

$$
\begin{aligned}
\mid \mathfrak{x}\left(u_{0}+\right. & \left.r e^{i \vartheta^{\prime \prime}}\right)-\left.\mathfrak{x}\left(u_{0}+r e^{i, \vartheta^{\prime}}\right)\right|^{2}=\left.\int_{\vartheta^{\prime}}^{\vartheta^{\prime \prime}} \mathfrak{x}_{\vartheta^{\prime}}\left(u_{0}+r e^{i, \vartheta}\right) d \vartheta\right|^{2} \\
& \leqq\left(\vartheta^{\prime \prime}-\vartheta^{\prime}\right) \int_{\vartheta^{\prime}}^{\vartheta^{\prime \prime}} \mathfrak{x}_{\vartheta}^{2}\left(u_{0}+r e^{i \vartheta}\right) d \vartheta \\
& \leqq r\left(\vartheta^{\prime \prime}-\vartheta^{\prime}\right) \frac{1}{2} \int_{\vartheta^{\prime}}^{\vartheta^{\prime \prime}}|\nabla \mathfrak{r}(r, \vartheta)|^{2} r d \vartheta \\
& \leqq \pi r \Phi^{\prime}(r)<2 \pi \gamma \Phi(r)<\varepsilon^{2}
\end{aligned}
$$

From these inequalities the following conclusions can be drawn: The limits

$$
\lim _{\vartheta \rightarrow+0} \mathfrak{x}\left(u_{0}+r e^{i \vartheta}\right)=\mathfrak{x}^{\prime}, \quad \lim _{\vartheta \rightarrow \pi-0} \mathfrak{x}\left(u_{0}+r e^{i \vartheta}\right)=\mathfrak{x}^{\prime \prime}
$$


exist. $\mathfrak{x}^{\prime}$ and $\mathfrak{x}^{\prime \prime}$ are points on the surface $T$. All points $\mathfrak{x}\left(u_{0}+r e^{i \vartheta}\right)$ for $0<\vartheta<\pi$ are contained in the ball $\left|\mathfrak{x}-\mathfrak{x}^{\prime}\right|<\varepsilon$ and, therefore, in the region $B\left(\mathfrak{x}^{\prime}, c ; \varepsilon\right)$. For the next estimates we shall use a distinguished coordinate system associated with the point $\mathfrak{x}^{\prime}$.

The comparison surface $\bar{S}=\left\{\mathfrak{x}=\overline{\mathfrak{x}}(w) ; w \in P^{\prime}\right\}$ with the position vector

$\overline{\mathfrak{x}}(w) \equiv \overline{\mathfrak{x}}(\varrho, \vartheta)= \begin{cases}{\left[R(r, \vartheta)+\frac{\varrho}{r} \lambda(r, \vartheta)\right] \mathfrak{N}(r, \vartheta)} & \text { for } w \in C\left(u_{0} ; r\right) \\ \mathfrak{x}(w) & \text { for } w \in P^{\prime \backslash C}\left(u_{0} ; r\right)\end{cases}$

obviously is bounded by the chain $\langle T, T\rangle$. The vector $\overline{\mathfrak{x}}(w)$ is linearly absolutely continuous in $P$. For $w \in C\left(u_{0} ; r\right)$ we have

$\overline{\mathfrak{x}}_{\varrho}(\varrho, \vartheta)=\frac{1}{r} \lambda(r, \vartheta) \mathfrak{N}(r, \vartheta)$,

$\overline{\mathfrak{x}}_{\vartheta}(\varrho, \vartheta)=\left[R_{\vartheta}(r, \vartheta)+\frac{\varrho}{r} \lambda_{\vartheta}(r, \vartheta)\right] \mathfrak{X}(r, \vartheta)+\left[R(r, \vartheta)+\frac{\varrho}{r} \lambda(r, \vartheta)\right] \mathfrak{N}_{\vartheta}(r, \vartheta)$

so that

$\overline{\mathfrak{x}}_{g}(\varrho, \vartheta) \times \overline{\mathfrak{x}}_{\vartheta}(\varrho, \vartheta)=\frac{1}{r} \lambda(r, \vartheta)\left[R(r, \vartheta)+\frac{\varrho}{r} \lambda(r, \vartheta)\right] \mathfrak{A}(r, \vartheta) \times \mathfrak{P}_{\vartheta}(r, \vartheta)$.

Now

$$
\begin{aligned}
\left|\mathfrak{R}(r, \vartheta) \times \mathfrak{P}_{\vartheta \vartheta}(r, \vartheta)\right| & \leqq \mathfrak{T}_{\vartheta}(r, \vartheta)|\leqq r| \nabla \mathfrak{l}(r, \vartheta) \mid \\
& \leqq \frac{r}{|\mathfrak{x}|}|\nabla \mathfrak{x}(r, \vartheta)| \leqq \frac{r}{1-\varepsilon}|\nabla \mathfrak{x}(r, \vartheta)|
\end{aligned}
$$

and, since $\lim _{\vartheta \rightarrow+0} \lambda(r, \vartheta)=0$,

$$
\begin{aligned}
|\lambda(r, \vartheta)| & =\left|\int_{0}^{\vartheta} \lambda_{\vartheta}(r, \vartheta) d \vartheta\right| \\
& \leqq r \int_{0}^{\vartheta}|\nabla \lambda(r, \vartheta)| d \vartheta \\
& \leqq \frac{r}{\sqrt{2}}\left(1+\frac{2 c}{1-\varepsilon}\right) \int_{0}^{i}|\nabla \mathfrak{x}(r, \vartheta)| d \vartheta
\end{aligned}
$$

Finally, since $R(r, \vartheta) \leqq 1+\sqrt{2} c \varepsilon$ and $c<1 / 2$, 


$$
\begin{aligned}
\left|R(r, \vartheta)+\frac{\varrho}{r} \lambda(r, \vartheta)\right| & \leqq \frac{\varrho}{r}|R(r, \vartheta)+\lambda(r, \vartheta)|+\frac{r-\varrho}{r} R(r, \vartheta) \\
& \leqq \frac{\varrho}{r}|\mathfrak{x}(r, \vartheta)|+\frac{r-\varrho}{r}(1+\sqrt{2} c \varepsilon) \\
& \leqq \frac{\varrho}{r}(1+\varepsilon)+\frac{r-\varrho}{r}(1+\sqrt{2} c \varepsilon) \\
& \leqq 1+\varepsilon .
\end{aligned}
$$

Therefore

$$
\left|\overline{\mathfrak{x}}_{\varrho} \times \overline{\mathfrak{x}}_{\vartheta}\right| \leqq \frac{r}{\sqrt{2}} \frac{1+\varepsilon}{1-\varepsilon}\left(1+\frac{2 c}{1-\varepsilon}\right)|\nabla \mathfrak{x}(r, \vartheta)| \int_{0}^{\vartheta}|\nabla \mathfrak{x}(r, \vartheta)| d \vartheta
$$

and

$$
\begin{aligned}
\int_{0}^{\pi}\left|\overline{\mathfrak{x}}_{\varrho} \times \overline{\mathfrak{x}}_{\vartheta}\right| d \vartheta & \leqq \frac{r}{2 \sqrt{2}} \frac{1+\varepsilon}{1-\varepsilon}\left(1+\frac{2 c}{1+\varepsilon}\right)\left(\int_{0}^{\pi}|\nabla \mathfrak{x}(r, \vartheta)| d \vartheta\right)^{2} \\
& \leqq \frac{\pi}{2 \sqrt{2}} \frac{1+\varepsilon}{1-\varepsilon}\left(1+\frac{2 c}{1-\varepsilon}\right) \int_{0}^{\pi}|\nabla \mathfrak{r}(r, \vartheta)|^{2} r d \vartheta \\
& \leqq \frac{\pi}{\sqrt{2}} \frac{1+\varepsilon}{1-\varepsilon}\left(1+\frac{2 c}{1-\varepsilon}\right) \Phi^{\prime}(r) .
\end{aligned}
$$

Another integration yields

$$
\int_{0}^{r} d \varrho \int_{0}^{\pi}\left|\overline{\mathfrak{x}}_{\varrho} \times \overline{\mathfrak{x}}_{\vartheta}\right| d \vartheta \leqq \frac{\pi}{\sqrt{2}} \frac{1+\varepsilon}{1-\varepsilon}\left(1+\frac{2 c}{1-\varepsilon}\right) r \Phi^{\prime}(r) .
$$

Now

$$
\begin{gathered}
A(S)=D_{P \backslash C\left(u_{0} ; r\right)}[\mathfrak{x}]+\Phi(r) \\
A(\bar{S})=D_{P \backslash C\left(u_{0} ; r\right)}[\mathfrak{x}]+\int_{0}^{r} d \varrho \int_{0}^{\pi} \overline{\mathfrak{x}}_{\varrho} \times \overline{\mathfrak{x}}_{i t} \cdot d \theta .
\end{gathered}
$$

From the minimizing property of the surface $S$ it follows that

$$
\Phi(r) \leqq \frac{\pi}{\sqrt{2}} \frac{1+\varepsilon}{1-\varepsilon}\left(1+\frac{2 c}{1-\varepsilon}\right) r \Phi^{\prime}(r)
$$

or, remembering the definition of $c$ and $\varepsilon$,

$$
2 \gamma \Phi(r) \leqq r \Phi^{\prime}(r)
$$


This is a contradiction to our initial assumption $r \Phi^{\prime}(r)<2 \lambda \Phi(r)$. Thus we have to abandon this assumption. Rather, the inequality

$$
2 \gamma \Phi(r) \leqq r \Phi^{\prime}(r)
$$

must hold for almost all $r$ in $v_{0}<r<v_{0}+\delta$.

Integrating this differential inequality between the limits $r=v_{0}+\varrho$ and $r=v_{0}+\delta$, we obtain

$$
\Phi\left(v_{0}+\varrho\right) \leqq\left(\frac{v_{0}+\varrho}{v_{0}+\delta}\right)^{2 \gamma} \Phi\left(v_{0}+\delta\right) \leqq\left(\frac{2 \varrho}{\delta}\right)^{2 \gamma} D_{P}[\mathfrak{x}]
$$

and therefore

$$
D\left[\mathfrak{x} ; w_{0}, \varrho\right] \leqq D\left[\mathfrak{x} ; u_{0}, v_{0}+\varrho\right] \leqq\left(\frac{2 \varrho}{\delta}\right)^{2 \gamma} D_{P}[\mathfrak{X}] \quad \text { for } \quad v_{0}<\varrho<\delta .
$$

We have now dealt with the cases $v_{0} \geqq \delta$ and $0<v_{0}<\varrho<\delta$. For the last case $0<\varrho \leqq v_{0}<\delta$ a combination of the preceding inequalities gives

$$
\begin{aligned}
D\left[\mathfrak{x} ; w_{0}, \varrho\right] & \leqq\left(\frac{\varrho}{v_{0}}\right)^{2 \gamma} D\left[\mathfrak{x} ; w_{0}, v_{0}\right] \leqq\left(\frac{\varrho}{v_{0}}\right)^{2 \gamma} D\left[\mathfrak{x} ; u_{0}, 2 v_{0}\right] \\
& \leqq\left(\frac{\varrho}{v_{0}}\right)^{2 \gamma}\left(\frac{2 v_{0}}{\delta}\right)^{2 \gamma} D_{P}[\mathfrak{x}] \leqq\left(\frac{2 \varrho}{\delta}\right)^{2 \gamma} D_{P}[\mathfrak{x}]
\end{aligned}
$$

Setting $M=(2 / \delta)^{2 \gamma} D_{P}[\mathfrak{x}]$, we have in all cases

$$
D\left[\mathfrak{x} ; w_{0}, \varrho\right] \leqq M \varrho^{2 \gamma} \quad \text { for } \quad w_{0} \in P_{r}, \quad 0<\varrho<\delta .
$$

Our assertion and, with it, theorem 1 is proved.

Acknowledgement. The preceding research was sponsored by the Air Force Office of Scientific Research under AFOSR Grant No. 883-67.

University of Minnesota

Minneapolis, Minnesota 55455, USA 


\section{Bibliography}

[1] Carathéodory, C.: Conformal representation. - 2nd edition, Cambridge Tracts in Mathematics and Mathematical Physics 28, Cambridge University Press, Cambridge, 1952.

[2] Cesari, L.: Surface area. - Annals of Mathematics Studies 35, Princeton University Press, Princeton (N.J.), 1956.

[3] Courant, R.: The existence of a minimal surface of least area bounded by prescribed Jordan arcs and prescribed surfaces. - Proc. Nat. Acad. Sci. U.S.A. 24, 1938, pp. 97-101.

[4] - - The existence of minimal surfaces of given topological structure under prescribed boundary conditions. - Acta Math. 72, 1940, pp. 51-98.

[5] - - On Plateau's problem with free boundaries. - Proc. Nat. Acad. Sci. U.S.A. 31,1945 , pp. $242-246$.

[6] -»- Dirichlet's principle, conformal mapping, and minimal surfaces. - Pure and Applied Mathematics 3, Interseience Publishers, New York/ London, 1950.

[7] Courant, R., and N. Davids: Minimal surfaces spanning closed manifolds. Proc. Nat. Acad. Sci. U.S.A. 26, 1940, pp. 194-199.

[8] Davids, N.: Minimal surfaces spanning closed manifolds and having prescribed topological position. - Amer. J. Math. 64, 1942, pp. 348-362.

[9] Douglas, J.: Solution of the problem of Plateau. - Trans. Amer. Math. 33, 1931, pp. $263-321$.

[10] Hildebrandt, S.: Über Minimalflächen mit freiem Rand. - Math. Z. 95, 1967, pp. 1-19.

[11] JäGER, W.: Behavior of minimal surfaces with free boundaries. - Comm. Pure Appl. Math. 23, 1970, pp. 803-818.

[12] Lebesgue, H.: Sur le problème de Dirichlet. - Rend. Circ. Mat. Palermo 24, 1907 , pp. $371-402$.

[13] Lewy, H.: On minimal surfaces with partially free boundary. - Comm. Pure Appl. Math. 4, 1951, pp. 1-13.

[14] McShane, E. J.: Integrals over surfaces in parametric form. - Ann. of Math. (2) 34,1933 , pp. $815-838$.

[15] - - Parametrizations of saddle surfaces with applications to the problem of Plateau. - Trans. Amer. Math. Soc. 35, 1933, pp. 716-733.

[16] Morrey, C. B., Jr.: A class of representations of manifolds. I. - Amer. J. Math. 55,1933, pp. $683-707$.

[17] -》- A class of representations of manifolds. II. - Amer. J. Math. 56, 1934, pp. $275-293$.

[18] -»- An analytic characterization of surfaces of finite Lebesgue area. I. Amer. J. Math. 57, 1935, pp. 692-702.

[19] - - An analytic characterization of surfaces of finite Lebesgue area. II. Amer. J. Math. 58, 1936, pp. 313-322. 
[20] Morrey, C. B., Jr.: On the solutions of quasi-linear elliptic partial differential equations. - Trans. Amer. Math. Soc. 43, 1938, pp. 126-166.

[21] Nitsche, J. C. C.: Variational problems with inequalities as boundary conditions, or, How to fashion a cheap hat for Giacometti's brother. - Arch. Rat. Mech. Anal. 35, 1969, pp. 83-113.

[22] - - - The behavior of minimal surfaces with free boundaries. - Rend. Circ. Mat. Palermo (2) 18, 1969, pp. 215-224.

[23] Pólya, G., and G. Szegö: Aufgaben und Lehrsätze aus der Analysis. I. - 2nd edition, Grundlehren der mathematischen Wissenschaften 19, SpringerVerlag, Berlin / Göttingen / Heidelberg, 1954.

[24] RADó, T.: On the problem of Plateau. - Ergebnisse der Mathematik und ihrer Grenzgebiete 2:2, Verlag von Julius Springer, Berlin, 1933.

[25] -》- Length and area. - American Mathematical Society Colloquium Publications 30, American Mathematical Society, New York, 1948.

[26] Ritter, I. F.: Solution of Schwarz' problem concerning minimal surfaces. - Univ. Nac. Tucumán Rev. Ser. A 1, 1940, pp. 49-62.

[27] Schwarz, H. A.: Ceber einen Grenzübergang durch alternierendes Verfahren. Gesammelte Mathematische Abhandlungen. II. Verlag von Julius Springer, Berlin, 1890, pp. 133-143. ( = Vierteljschr. Naturforsch. Ges. Zürich 15, 1870, pp. $272-286$.)

[28] Tonelti, L.: Sul problema di Plateau. I. - Opere scelte. III. E 'izioni Cremonese, Roma, 1962, pp. 328-335. (= Atti Reale Accad. Naz. Lincei. Rend. Cl. Sci. Fis. Mat. Nat. (6) 24, 1936, pp. 333-339.) 